\title{
c-kit and bcl-2 Are Not Useful Markers in Differentiating Adenoid Cystic Carcinoma from Polymorphous Low-Grade Adenocarcinoma
}

\author{
Shabnum Meer, ${ }^{1}$ Sunita Singh, ${ }^{2}$ and Mario Altini ${ }^{1}$ \\ ${ }^{1}$ Division of Oral Pathology, Faculty of Health Sciences, University of the Witwatersrand, Private Bag 3, Wits, \\ 2050 Johannesburg, South Africa \\ ${ }^{2}$ Division of Maxillofacial and Oral Surgery, Faculty of Health Sciences, University of the Witwatersrand, \\ 2050 Johannesburg, South Africa
}

Correspondence should be addressed to Shabnum Meer, shabnum.meer@nhls.ac.za

Received 28 April 2011; Accepted 24 May 2011

Academic Editors: A. Navarro and H.-U. Schildhaus

Copyright ( $) 2011$ Shabnum Meer et al. This is an open access article distributed under the Creative Commons Attribution License, which permits unrestricted use, distribution, and reproduction in any medium, provided the original work is properly cited.

\begin{abstract}
Aims. To evaluate the immunohistochemical expression of bcl-2 and c-kit proteins in the salivary gland tumors, adenoid cystic carcinoma, and polymorphous low-grade adenocarcinoma with a view to tumor differentiation. Methods. Immunohistochemical studies were performed on 29 adenoid cystic carcinoma and 23 polymorphous low-grade adenocarcinoma archival cases with bcl-2 and c-kit antibodies using standard procedures, and the data were statistically analyzed. Results. The difference in bcl-2 expression in adenoid cystic carcinoma and polymorphous low-grade adenocarcinoma was not statistically significant. Whilst the difference in c-kit expression was statistically significant $(P=0.01)$, the difference proved small so as to be of no practical or diagnostic importance. Moderate to strong immunostaining was noted in adenoid cystic carcinoma with c-kit, the pattern and intensity varying within different histological subtypes, being greater the more aggressive the tumor subtype. Conclusions. Bcl-2 and c-kit are not useful as immunohistochemical markers in differentiating adenoid cystic carcinoma from polymorphous low-grade adenocarcinoma.
\end{abstract}

\section{Introduction}

Adenoid cystic carcinoma (ACC) and polymorphous lowgrade adenocarcinoma (PLGA) are salivary gland malignancies with some overlapping histomorphologic features and immunophenotypic profiles, yet their biologic behavior is significantly different. Some histopathologic similarity is expected as both tumors are composed of ductal and abluminal myoepithelial differentiated cells. Both tumors have a marked propensity to infiltrate around nerves. The distinction between ACC and PLGA is important because ACC is clinically more aggressive and infiltrative, requiring a more radical surgical approach. Although these tumors are often readily diagnosed, occasionally differentiation may pose a diagnostic challenge especially when examining tissue from small incision or fragmented biopsies [1]. There remains a need for better-defined immunohistochemical distinction between ACC and PLGA.

Studies [1-3] have alluded to the role of c-kit and bcl2 overexpression as potential immunomarkers in ACC and PLGA. c-kit is a $145-160 \mathrm{kDa}$ transmembrane tyrosine kinase receptor, encoded by c-kit proto-oncogene, and normally expressed in hematopoietic stem cells, mast cells, interstitial cells of Cajal, melanocytes, basal cells of skin, breast epithelial cells, germ cells, and cells of the central nervous system $[2,3]$. Overexpression or de novo expression has been observed, amongst others, in renal, prostate, and ovarian cancer, gastrointestinal stromal tumors, gliomas, neuroblastomas, and seminomas suggesting a role for c-kit receptor and mutations of its gene in carcinogenesis [3]. The oncoprotein, bcl-2, which has the ability to downregulate cell death by apoptosis allowing cells to survive even in the absence of essential 
growth factors, is expressed in a variety of normal and diseased tissues including neuroblastoma, follicular lymphoma, pulmonary, breast, prostate, and nasopharyngeal carcinoma [4].

Although overexpression of both proteins is well documented in many body tissues and tumor types expression in salivary gland neoplasia is limited, and investigations in the use of c-kit in ACC and PLGA have yielded incongruous results [2-7]. The unusual overexpression of c-kit in salivary ACC compared to other head and neck tumors suggests ckit expression as a diagnostic pathology aid for ACC [8]. The simple immunohistochemical assessment of ACC and PLGA does not surpass careful assessment of morphologic features. Determination of the differential expression of c-kit and bcl2 in ACC and PLGA may be a potentially useful adjunct in the distinction between the tumors. The aim of this study was thus to determine and compare the immunoexpression of bcl-2 and c-kit (CD117) proteins in ACC and PLGA with a view to using these markers in their differentiation.

\section{Methods}

2.1. Biopsy Material. 29 cases of ACC (Figure 1(a)) and 22 cases of PLGA (Figure 1(b)) were accessioned from the archives of the Division of Oral Pathology, University of the Witwatersrand, from the period 1997 to 2003. Hematoxylin and eosin (H\&E) stained sections were independently reviewed by 2 experienced pathologists and the diagnoses confirmed. All tissue specimens were originally fixed in $10 \%$ neutral buffered formalin (18-48 hours), routinely processed, and embedded in paraffin wax. The ethics clearance number, M00/08/29, for this study was issued by the Human Research Ethics Committee (Medical) of the University of the Witwatersrand, Johannesburg, for use of archival block material obtained from human tissues.

2.2. Immunohistochemistry. Immunohistochemistry was performed on deparaffinized $4 \mu \mathrm{m}$ sections with monoclonal bcl-2 oncoprotein (NCL-bcl-2-486, clone 3.1, Novocastra, Newcastle upon Tyne, UK) and polyclonal c-kit (CD117) oncoprotein (rabbit anti-CD117 antibody, code A4502, DakoCytomation A/S, Glostrup, Denmark) against the carboxyl terminus of human c-kit amino acids 963 to 976. A pilot study demonstrated that the latter antibody showed more consistent and intense staining than monoclonal c-kit (CD117) oncoprotein (NCL-CD117 clone T595, Novocastra, Newcastle upon Tyne, UK) against the $\mathrm{N}$-terminal $\mathrm{O}_{2}$ like extracellular domain of human c-kit. The $4 \mu \mathrm{m}$ sections were placed on 3-aminopropyl-triethoxysilane (APES, Sigma, St. Louis, MO) glass slides, which were air dried overnight, dewaxed, and hydrated through graded alcohols and water. The sections were pretreated by heating in a microwave oven ( 800 watt) on medium power for 10 minutes. The bcl-2 antibody was microwaved in citrate buffer at $\mathrm{pH}$ 6.0, while for c-kit microwave heat-induced epitope retrieval was performed in Tris buffer EDTA (TBST) at pH 9.0. Sections were cooled for 20 minutes. Biotin blocking (5-15 minutes) was carried out for bcl-2 but not for c-kit. This was achieved by incubating with avidin for 10 minutes then rinsed with TBST and incubated for 3 minutes. It was then incubated with biotin for 10 minutes, rinsed with TBST, and incubated for 3 minutes.

The sections were immersed in 3\% hydrogen peroxide in distilled water for 5 minutes and rinsed in TBS pH 7.6 with $0.1 \%$ Tween 20 (Sigma, St. Louis, MO). Specimens were incubated with the primary antibodies using the following dilutions: 1:50 for bcl-2 and 1:100 for c-kit. Chromogen was applied for 5 minutes, and the color developed with diaminobenzidine hydrochloride (DAB, Sigma, St. Louis, $\mathrm{MO}$ ) resulting in a brown reaction product. Sections were then lightly counterstained with $\mathrm{H} \& \mathrm{E}$ for 1 minute. Appropriate positive controls, tonsil for bcl-2 and skin for c-kit, were used, along with the omission of the primary antibodies as negative controls.

2.3. Evaluation. Dense brown nuclear and cytoplasmic staining of the tumor cells was regarded as positive for each antibody. Both membranous and cytoplasmic c-kit staining patterns were included in the analyses. As reported previously [9], the intensity of staining was graded as weak, moderate, or intense. The sum of the staining intensity was used as the total immunoreactivity. Immunoevaluation included enumeration of 500 cells from 5 fields as the percentage of reactive cells. The estimated percentage of positive cells based on the consensus of 2 observers examining the entire tissue field on each section at low- $(\times 4$ objective $)$ and high-power magnification $(\times 40$ objective $)$ was categorized as $<30 \%$, $30-60 \%$, and $>60 \%$. The chi-squared test was used to test whether there was a statistical difference between ACC and PLGA for each antibody. For all tests, a $P$ value $=0.05$ was chosen as the critical level for statistical significance. Intra- and interexaminer reliability for the bcl-2 and ckit assessment was achieved by the same examiner scoring all cases one week later, and then 2 different examiners, respectively, giving an independent overall assessment and score of each case of ACC and PLGA and comparing the results using a Student $t$-test.

\section{Results}

3.1. Clinicopathology. The clinical and histological features of both ACC (Figure 1(a)) and PLGA (Figure 1(b)) are outlined in Table 1 . The histological subtypes of the 29 cases of ACC included cribriform $(n=16)(55.2 \%)$, tubular $(n=$ $3)(10.3 \%)$, solid $(n=3)(10.3 \%)$, and mixed $(n=7)$ (24.1\%) patterns.

3.2. Immunohistochemistry. Statistical analysis of the interand intraobserver readings clearly established the reliability and reproducibility of the assessment of immunostaining of both antibodies used and of the data obtained. The results of the paired sample $t$-test showed no statistically significant difference between the data; $P$ was always $>0.05$ for the interand intraobserver comparison (bcl-2 $P=0.02$; c-kit $P=$ $0.03)$. 


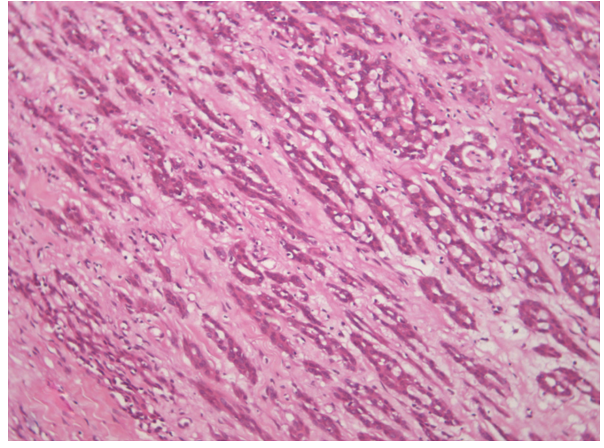

(a)

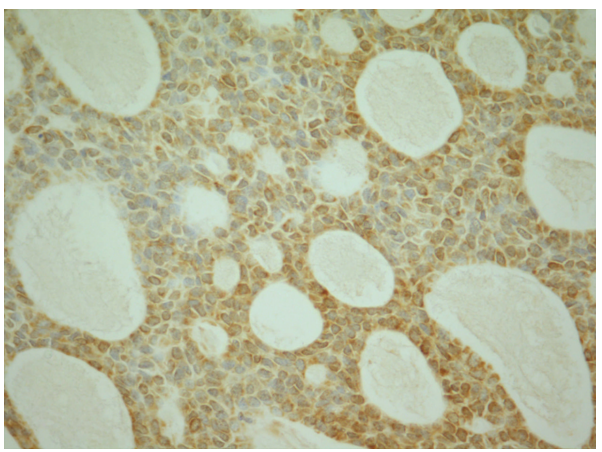

(c)

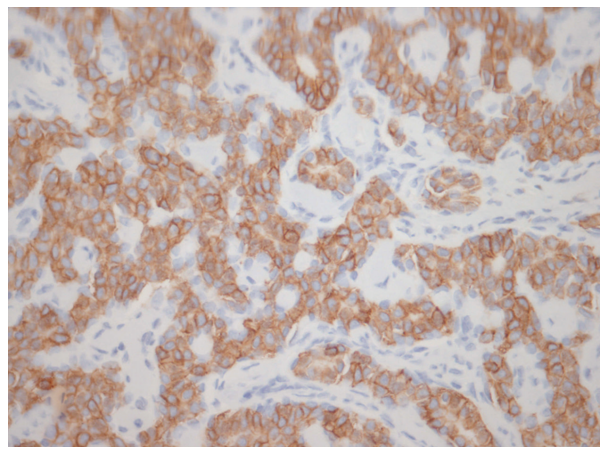

(e)

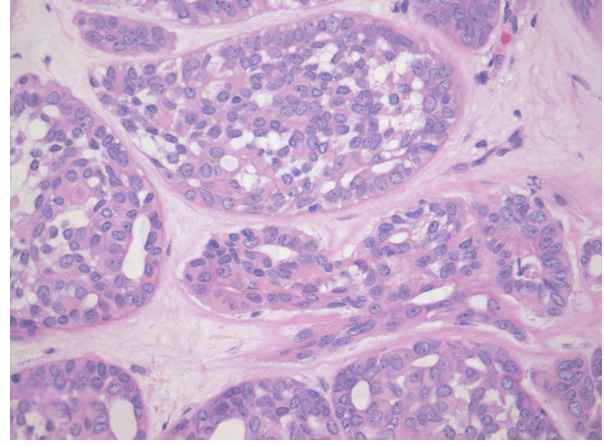

(b)

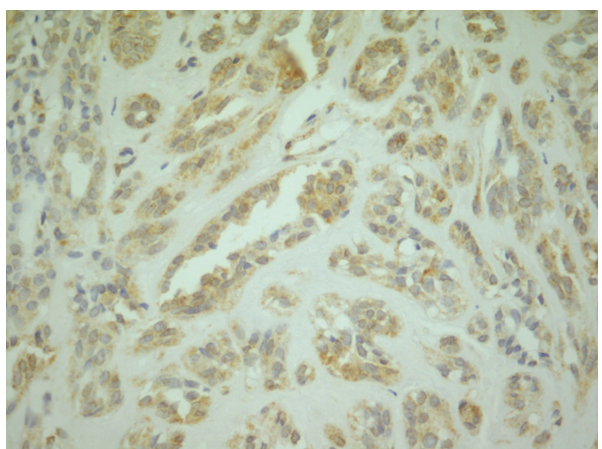

(d)

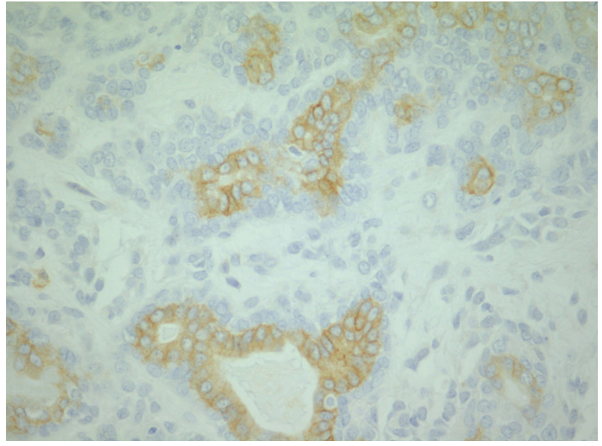

(f)

FIgURE 1: Staining patterns in ACC and PLGA. ACC showing a focal cribriform growth pattern as well as areas of streaming mimicking a PLGA (a) (H\&E, $\times 20)$ and a PLGA with biphasic structures resembling ACC (b) (H\&E, $\times 40)$. Marked (>60\%) granular cytoplasmic bcl-2 protein expression in the cribriform variant of ACC (c) and the tubular type of PLGA (d) (bcl-2, $\times 40)$. Intense c-kit membranous and cytoplasmic immunopositivity ( $>60 \%)$ of the luminal cells in ACC (e); weak $(<30 \%)$ cytoplasmic and membranous staining in PLGA (f) (c-kit, $\times 40)$.

Immunoexpression of bcl-2 and c-kit is summarized in Table 2. Bcl-2 was moderately to strongly expressed (30$60 \%$ and $>60 \%)$ in $23 / 29(79.3 \%)$ of ACCs showing granular cytoplasmic staining (Figure 1(c)) and in 19/23 (82.6\%) of PLGAs (Figure 1(d)). Moderate to intense c-kit immunoexpression $(30-60 \%$ and $>60 \%)$ was noted in $16 / 29(55.2 \%)$ cases of ACC with decreased expression within the 4/23 (17.4\%) PLGA cases (Figures 1(e) and 1(f)). C-kit was expressed in all the neoplastic cells in solid ACC and in the inner cell layers, and the luminal cells of the tubular and cribriform types. The intensity of immunostaining was the strongest in the cribriform type (Figure 1(e)). In PLGA, the overall staining intensity appeared weaker than that of ACC, with membrane staining and a cytoplasmic blush typically seen. C-kit expression was noted in the luminal and nonluminal cells of the ductal, trabecular, and lobular structures (Figure 1(f)).

The results of the pilot study with c-kit monoclonal antibody showed moderate to intense positivity in $10.3 \%$ of ACC and $4.3 \%$ of PLGA, with no significant difference in staining expression or intensity between the tumors. This was different to the c-kit polyclonal antibody which was 
TABLe 1: Clinical findings in ACC and PLGA.

\begin{tabular}{|c|c|c|c|c|}
\hline \multirow{3}{*}{ Total number of cases } & \multicolumn{2}{|c|}{$\mathrm{ACC}$} & \multicolumn{2}{|c|}{ PLGA } \\
\hline & \multicolumn{2}{|c|}{29} & \multicolumn{2}{|c|}{22} \\
\hline & $n$ & $\%$ & $n$ & $\%$ \\
\hline Primary & 26 & 89.7 & 22 & 100 \\
\hline Recurrent & 3 & 10.3 & 0 & 0 \\
\hline \multicolumn{5}{|l|}{ Site of involvement } \\
\hline Major glands & 11 & 37.9 & 0 & 0 \\
\hline Parotid & 3 & 10.3 & & \\
\hline Submandibular & 8 & 27.6 & & \\
\hline Minor glands & 17 & 58.6 & 22 & 100 \\
\hline Palate & 10 & 34.5 & 15 & 68.2 \\
\hline Maxilla & 4 & 13.8 & 2 & 9.1 \\
\hline Buccal mucosa & 1 & 3.4 & 2 & 9.1 \\
\hline Floor of mouth & 1 & 3.4 & 0 & 0 \\
\hline Tongue & 1 & 3.4 & 1 & 4.5 \\
\hline Upper lip & 0 & 0 & 2 & 9.1 \\
\hline Unknown & 1 & 3.4 & & \\
\hline Major: minor glands & \multicolumn{2}{|c|}{$1: 1.6$} & & \\
\hline \multicolumn{5}{|l|}{ Gender } \\
\hline Male & 7 & 24.1 & 8 & 36.4 \\
\hline Female & 22 & 75.9 & 14 & 63.6 \\
\hline Male : female & \multicolumn{2}{|c|}{$1: 3.1$} & \multicolumn{2}{|c|}{$1: 1.8$} \\
\hline \multicolumn{5}{|l|}{ Age in years } \\
\hline Mean & \multicolumn{2}{|c|}{46.6} & \multicolumn{2}{|c|}{57.8} \\
\hline Median & \multicolumn{2}{|c|}{43} & \multicolumn{2}{|c|}{58} \\
\hline Range & \multicolumn{2}{|c|}{$22-74$} & \multicolumn{2}{|c|}{$23-77$} \\
\hline \multicolumn{5}{|l|}{ Race } \\
\hline Black & 27 & 93.1 & 19 & 86.4 \\
\hline White & 1 & 3.4 & 3 & 13.6 \\
\hline Indian & 1 & 3.4 & 0 & 0 \\
\hline
\end{tabular}

expressed in $55.2 \%$ of ACC and in $17.4 \%$ of PLGA. The expression of c-kit staining in both ACC and PLGA was decreased in comparison to c-kit (polyclonal) staining.

The overall distribution of different staining intensities with bcl-2 and c-kit in both ACC and PLGA, analyzed by chisquared testing, showed a small yet statistically significantly increased moderate to intense staining in ACC, with weaker staining in PLGA $(P=0.01)$. Even though this result may be statistically significant, it appears that the difference is small enough so as to be utterly insignificant. Staining intensity of bcl-2 and c-kit (monoclonal) antibodies showed no statistical difference.

\section{Discussion}

A number of studies have attempted to differentiate ACC from PLGA via the use of antibodies to GFAP, vimentin, S100 protein, Ki-67 (MIB-1), $\alpha$-smooth muscle actin, musclespecific actin, cytokeratins, intergrins, c-kit, bcl-2, cadherins, and CD43 $[1,10]$. No single antibody has yet been identified that can unequivocally be used in the differentiation of ACC and PLGA.
This study demonstrated a statistically significant decrease in staining intensity with c-kit in PLGA when compared to ACC. Although many studies have reported c-kit to be a useful ancillary marker in the diagnosis of ACC and in distinguishing ACC from PLGA, the results of detectable ckit expression in salivary gland neoplasia remain ambiguous $[1,2,6,9,11]$. Beltran et al. [11] demonstrated a statistically significant difference $(P \leq 0.001)$ in c-kit expression between the tumors, with $100 \%$ c-kit immunoreactivity in ACC and no expression in $80 \%$ of PLGA. Others reported greater positive cytoplasmic c-kit expression in ACCs ( $>50 \%$ in $80-$ $83 \%$ of cases $)$, with no or minimal staining in PLGA $(<50 \%$ in $41 \%$ of cases) $[2,3,9]$.

We referred previously to a pilot study in which it was determined that the 2 types of c-kit (monoclonal and polyclonal) resulted in dissimilar staining. The discrepancies in the specificity of c-kit staining are reported to be due to the variability of the primary antibodies selected and the influence of factors such as differences in immuno histochemical protocols (including deparaffination, epitope retrieval methods, dilutions, detection reagents used, and immunohistochemical methods), the varying methods of evaluating 
TABLE 2: Total immunoreactivity of bcl-2 and c-kit observed in ACC and PLGA expressed as number of cases $(n)$ and as a percentage (\%).

\begin{tabular}{|c|c|c|c|c|c|c|c|c|c|c|c|c|}
\hline \multirow{2}{*}{ Total $n$ of cases } & \multicolumn{6}{|c|}{ ACC } & \multicolumn{6}{|c|}{ PLGA } \\
\hline & \multicolumn{6}{|c|}{29} & \multicolumn{6}{|c|}{23} \\
\hline \multirow{2}{*}{$\%$ immunostaining } & \multicolumn{2}{|c|}{$<30$} & \multicolumn{2}{|c|}{$30-60$} & \multicolumn{2}{|c|}{$>60$} & \multicolumn{2}{|c|}{$<30$} & \multicolumn{2}{|c|}{$30-60$} & \multicolumn{2}{|c|}{$>60 \%$} \\
\hline & $n$ & $\%$ & $n$ & $\%$ & $n$ & $\%$ & $n$ & $\%$ & $n$ & $\%$ & $n$ & $\%$ \\
\hline bcl-2 & 6 & 20.7 & 2 & 6.9 & 21 & 72.4 & 4 & 17.4 & 6 & 26.1 & 13 & 56.5 \\
\hline c-kit polyclonal & 13 & 44.8 & 8 & 27.6 & 8 & 27.6 & 19 & 82.6 & 2 & 8.7 & 2 & 8.7 \\
\hline c-kit monoclonal (pilot study) & 26 & 89.7 & 1 & 3.4 & 2 & 6.9 & 22 & 95.7 & 0 & 0 & 1 & 4.3 \\
\hline
\end{tabular}

immunoreactivity, and the limited number of cases reviewed $[3,9,11]$. In this study, c-kit staining in ACC was mainly cytoplasmic; however membranous, nuclear, and cytoplasmic staining was noted when the staining was more intense. As shown previously, c-kit expression was noted in the luminal cells of the tubular and cribriform structures, and the pseudocysts, with diffuse c-kit reactivity in all cell types of the solid variant of ACC $[1-3,9]$.

This study, unlike previous reports, showed no preference in c-kit expression for major or minor salivary glands in ACC $[1,12]$. Even though no c-kit staining in nonneoplastic salivary gland tissue and variable staining in ACC has been previously reported, this study, like others, showed occasional weak, focal positivity in the ducts of the adjacent nonneoplastic salivary tissue $[3,6,13]$. No mutation of the c-kit juxtamembranous domain (exon 11) and kinase domain 2 (exon 17) using polymerase chain reaction (PCR) and DNA sequencing was detected [6]. Furthermore, c-kit expression has been reported in acinic cell carcinoma (84\%), epithelialmyoepithelial carcinoma (100\%), carcinosarcoma (100\%), undifferentiated (lymphoepithelial) carcinoma (67\%), basal cell adenocarcinoma (100\%), and chronic sialadenitis with no expression in mucoepidermoid carcinoma, squamous cell carcinoma, salivary duct carcinoma, myoepithelial carcinoma, oncocytic carcinoma, and adenocarcinoma [3].

Like previous reports, the pattern and intensity of c-kit immunostaining in ACC in this study varied with the different histological subtypes, with the more aggressive tumors showing greater staining intensity $[6,9,13]$. The solid type showed diffuse reactivity compared to luminal expression in the cribriform and tubular variants. C-kit expression primarily in the luminal cell layer with no expression in the abluminal cells within the tubular and cribriform variants indicates that the myoepithelial cells do not express c-kit [13]. Ironically, solid variants show expression in all cells with most considered modified myoepithelial cells. The variable patterns and intensity of staining observed in ACC may be the result of a loss of cellular heterogeneity in the solid variant, with the differentiation primarily along the line of the luminal cell layer, and may correlate with the worse clinical course of the solid variant of ACC $[13,14]$.

Whilst the cribriform pattern and mixed subtypes exhibited higher c-kit reactivity than the tubular pattern alone, others found c-kit expression to be higher among solid and tubular subtypes than the cribriform subtype [7]. In contrast, Freier et al. [14] showed staining intensity of the solid type to be significantly lower than the cribriform and tubular subtypes, an unexpected finding since solid ACC is clinically more aggressive and may inherit a higher number of cytogenetic aberrations. Contrary to the study by Seethala et al. [15], this study suggests that c-kit staining is more frequently found in well-differentiated ACC tumors and is possibly lost during dedifferentiation, indicating the involvement of molecular pathways in the formation of the histological subtypes.

There was no statistically significant difference in bcl-2 expression between ACC and PLGA. ACC showed more intense, granular cytoplasmic and/or nuclear bcl-2 reactivity, with PLGAs exhibiting a weak to moderate cytoplasmic and membranous stain. Adjacent salivary gland ducts, lymphatic tissue, and nerves also expressed bcl-2. In the nonneoplastic salivary gland tissue, bcl-2 positivity was observed in ductal but not in acinar cells. Others have also reported bcl-2 expression in basal cells of striated and excretory ducts, indicating that these cells are reserve cells and that acinar, myoepithelial, and most luminal cells are negative for bcl-2 [5].

Whilst the expression of bcl-2 has been assessed in some salivary gland tumors, namely, pleomorphic adenoma, monomorphic adenoma, Warthin's tumor, basal cell adenoma, mucoepidermoid carcinoma, adenocarcinoma (NOS), acinic cell carcinoma, adenoid cystic carcinoma, anaplastic carcinoma, squamous cell carcinoma, and basal cell adenocarcinoma, its use as an immunohistochemical marker in the differentiation between ACC and PLGA has not been reported [16]. Bcl-2 reactivity in ACC was found to be the strongest when compared to acinic cell carcinoma, mucoepidermoid carcinoma, and undifferentiated carcinoma [5]. This was attributed to the fact that other tumor genes that influence apoptosis may be present in salivary gland tumors but bypass the bcl-2 associated apoptotic pathway; such genes include $\mathrm{p} 53, \mathrm{Rb}$ tumor suppressor genes, and c-myc proto-oncogene. Malignant salivary gland tumors were also found to often show no or weak bcl-2 positivity compared with the benign tumors.

A higher expression of bcl-2 was noted in the solid and cribriform types of ACC suggesting that bcl-2 expression might be associated with myoepithelial cells as these types have more myoepithelial than ductal cells [17]. Furthermore, a significant inverse correlation between bcl-2 reactivity and the apoptotic index $(P=0.018)$ was found supporting the suppressing mechanism of bcl-2 in the regulation of apoptosis. Several studies have indicated high immunoreactivity of bcl-2 in ACC, but no comparison to PLGA has been reported $[5,17]$. Based on the assumption that PLGA is of lower grade 
than ACC, one would expect a higher immunoreactivity to bcl-2 in PLGA. On the contrary, though, in our study the ACC tended to exhibit a more intense bcl-2 reactivity compared to PLGA.

The distinction between ACC and PLGA is important since the clinical course and prognostic significance differ. It is imperative that immunohistochemical stains used as adjuncts in histological-based diagnosis be simple, accurate, reliable, and reproducible. Whilst use of antibodies such as $\alpha$-SMA and CD43 has been suggested as aids in the differentiation of ACC and PLGA, there is as yet no one single antibody that can be used unequivocally in the diagnosis of these tumors, especially in small tissue samples, but rather a panel of immunohistochemical agents can be used as an adjunct to aid in their diagnosis.

C-kit activity may be necessary for tumor maintenance in ACC. However, ACC seems to proliferate despite inactivation of the c-kit signaling pathway; thus selective c-kit inhibition is probably not useful in ACC therapeutic development [8]. Although, the strong uniform expression of c-kit in ACC may suggest a potential role for c-kit inhibitors in the management of ACC, the effect of tyrosinase kinase inhibitor (TKI) therapy depends on the presence of activating KIT mutations, and thus the effect of TKI in the treatment of ACC appears at least questionable. Further studies remain to be undertaken in this regard.

\section{Conclusion}

Neither bcl-2 nor c-kit appears to be valuable as single or joint immunohistochemical markers in the diagnostic differentiation of ACC from PLGA.

\section{Conflict of Interests}

The authors declare that there is no conflict of interests.

\section{References}

[1] P. C. Edwards, T. Bhuiya, and R. D. Kelsch, "C-kit expression in the salivary gland neoplasms adenoid cystic carcinoma, polymorphous low-grade adenocarcinoma, and monomorphic adenoma," Oral Surgery, Oral Medicine, Oral Pathology, Oral Radiology, and Endodontics, vol. 95, no. 5, pp. 586-593, 2003.

[2] Y. M. Jeng, C. Y. Lin, and H. C. Hsu, "Expression of the c-kit protein is associated with certain subtypes of salivary gland carcinoma," Cancer Letters, vol. 154, no. 1, pp. 107-111, 2000.

[3] D. Andreadis, A. Epivatianos, A. Poulopoulos et al., "Detection of C-KIT (CD117) molecule in benign and malignant salivary gland tumours," Oral Oncology, vol. 42, no. 1, pp. 57-65, 2006.

[4] F. Pezzella and K. Gatter, "What is the value of bcl-2 protein detection for histopathologists?" Histopathology, vol. 26, no. 1, pp. 89-93, 1995.

[5] Y. Soini, U. Törmänen, and P. Pääkkö, "Apoptosis is inversely related to bcl-2 but not to bax expression in salivary gland tumours," Histopathology, vol. 32, no. 1, pp. 28-34, 1998.

[6] V. A. Holst, C. E. Marshall, C. A. Moskaluk, and H. F. Frierson Jr., "Kit protein expression and analysis of c-kit gene mutation in adenoid cystic carcinoma," Modern Pathology, vol. 12, no. 10, pp. 956-960, 1999.

[7] M. Mino, B. Z. Pilch, and W. C. Faquin, "Expression of KIT (CD117) in neoplasms of the head and neck: an ancillary marker for adenoid cystic carcinoma," Modern Pathology, vol. 16, no. 12, pp. 1224-1231, 2003.

[8] O. Tetsu, J. Phuchareon, A. Chou, D. P. Cox, D. W. Eisele, and R. C. K. Jordan, "Mutations in the c-kit gene disrupt mitogenactivated protein kinase signaling during tumor development in adenoid cystic carcinoma of the salivary glands," Neoplasia, vol. 12, no. 9, pp. 708-717, 2010.

[9] A. Epivatianos, A. Poulopoulos, I. Dimitrakopoulos et al., "Application of $\alpha$-smooth muscle actin and c-kit in the differential diagnosis of adenoid cystic carcinoma from polymorphous low-grade adenocarcinoma," Oral Oncology, vol. 43, no. 1, pp. 67-76, 2007.

[10] V. L. Woo, T. Bhuiya, and R. Kelsch, "Assessment of CD43 expression in adenoid cystic carcinomas, polymorphous lowgrade adenocarcinomas, and monomorphic adenomas," Oral Surgery, Oral Medicine, Oral Pathology, Oral Radiology and Endodontology, vol. 102, no. 4, pp. 495-500, 2006.

[11] D. Beltran, W. C. Faquin, G. Gallagher, and M. August, "Selective immunohistochemical comparison of polymorphous low-grade adenocarcinoma and adenoid cystic carcinoma," Journal of Oral and Maxillofacial Surgery, vol. 64, no. 3, pp. 415-423, 2006.

[12] G. M. Oprea, G. Bachowski, S. J. Lundeen et al., "C-kit (CD117) expression is higher in adenoid cystic carcinoma (ACC) arising in major salivary glands than in ACC arising in other locations," in United States and Canadian Academy of Pathology Annual Meeting, p. 111, Chicago, Ill, USA, 2002, [abstract \#93].

[13] C. R. Penner, A. L. Folpe, and S. D. Budnick, "C-kit expression distinguishes salivary gland adenoid cystic carcinoma from polymorphous low-grade adenocarcinoma," Modern Pathology, vol. 15, no. 7, pp. 687-691, 2002.

[14] K. Freier, C. Flechtenmacher, A. Walch et al., "Differential KIT expression in histological subtypes of adenoid cystic carcinoma (ACC) of the salivary gland," Oral Oncology, vol. 41, no. 9, pp. 934-939, 2005.

[15] R. R. Seethala, J. L. Hunt, Z. W. Baloch, V. A. LiVolsi, and E. L. Barnes, "Adenoid cystic carcinoma with high-grade transformation: a report of 11 cases and a review of the literature," American Journal of Surgical Pathology, vol. 31, no. 11, pp. 1683-1694, 2007.

[16] R. M. Nagler, H. Kerner, S. Ben-Eliezer, I. Minkov, and O. Ben-Itzhak, "Prognostic role of apoptotic, Bcl-2, c-erbB-2 and p53 tumor markers in salivary gland malignancies," Oncology, vol. 64, no. 4, pp. 389-398, 2003.

[17] L. Jia, R. L. Esguerra, X. Tang et al., "Prognostic value of apoptosis and apoptosis-associated proteins in salivary gland adenoid cystic carcinoma," Pathology International, vol. 54, no. 4, pp. 217-223, 2004. 


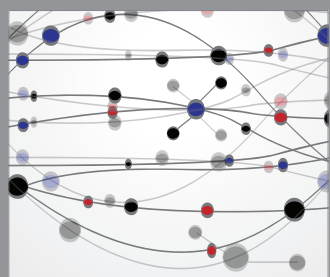

The Scientific World Journal
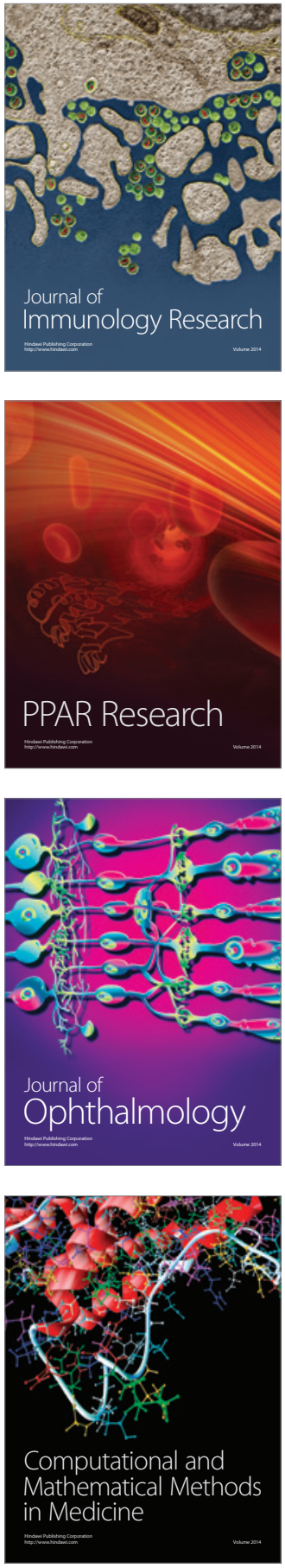

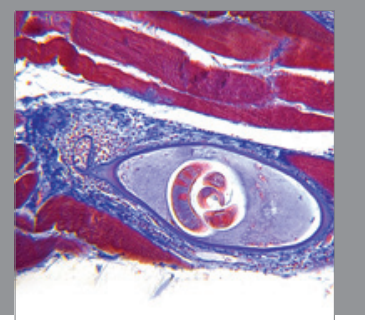

Gastroenterology

Research and Practice
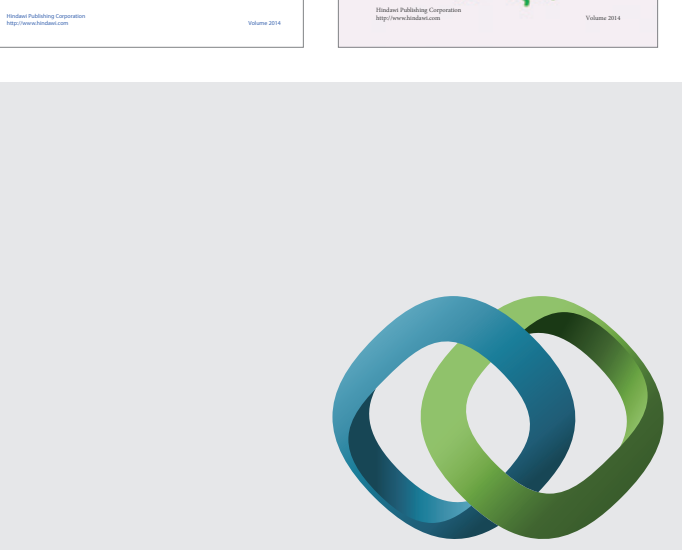

\section{Hindawi}

Submit your manuscripts at

http://www.hindawi.com
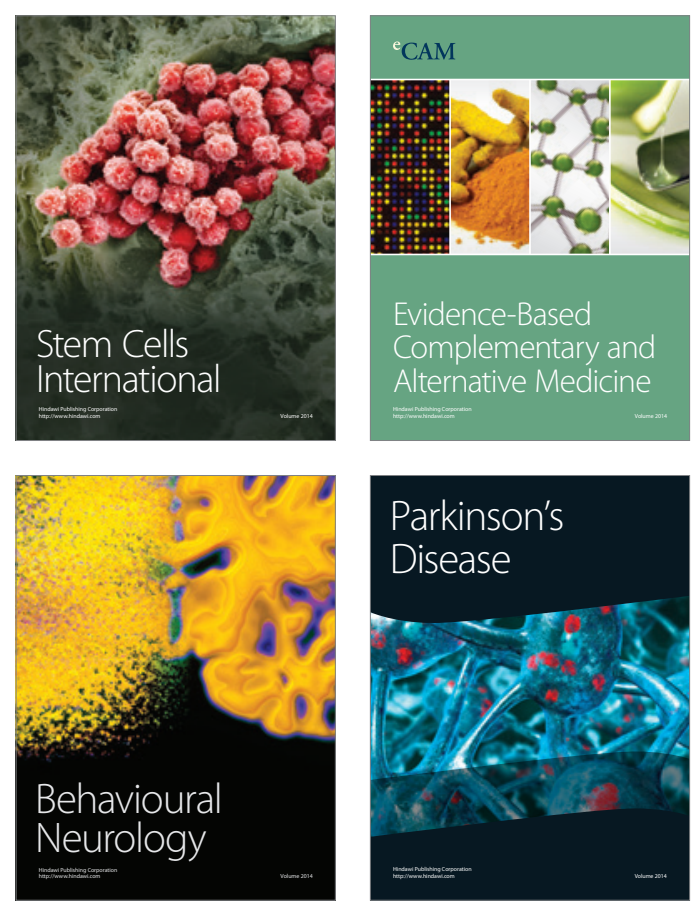

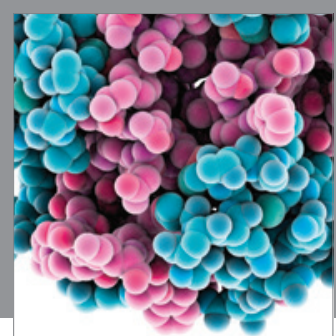

Journal of
Diabetes Research

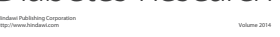

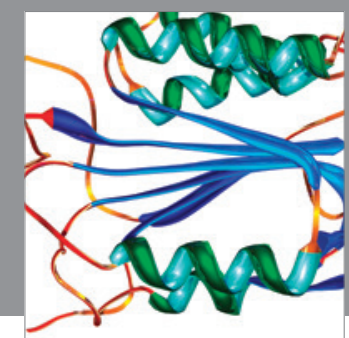

Disease Markers
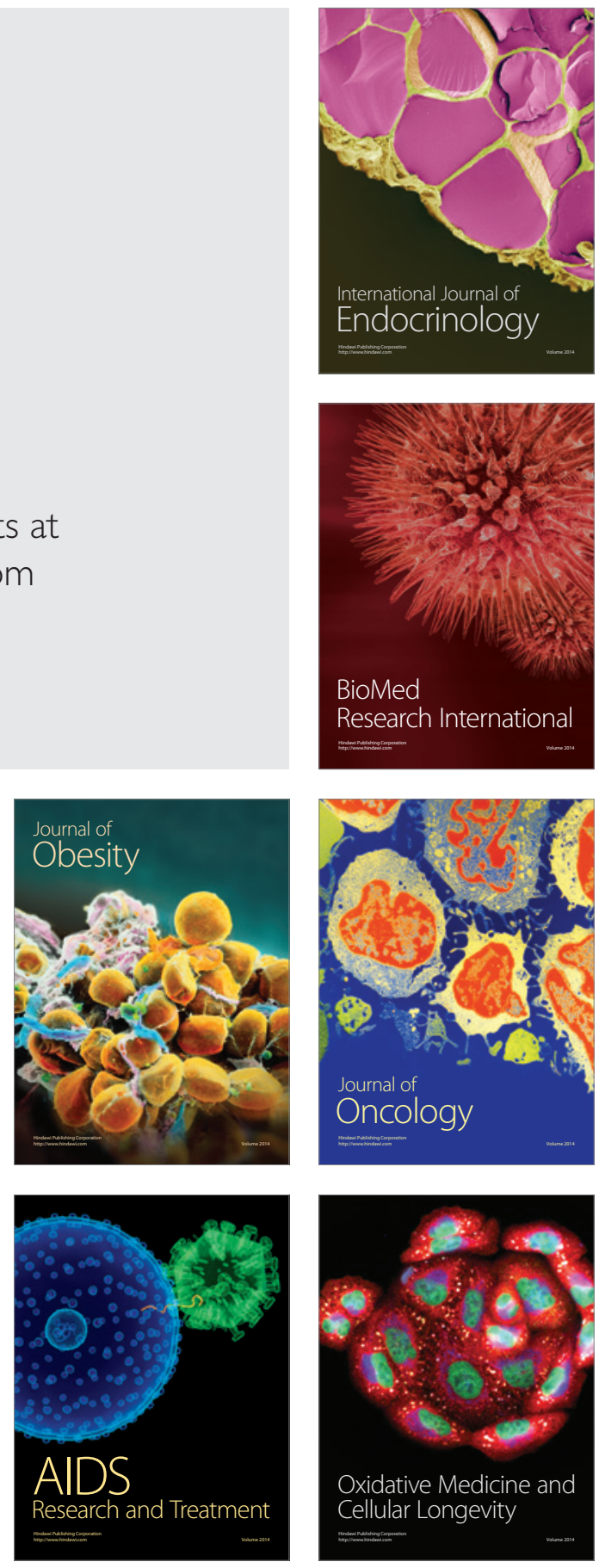